\title{
Loop corrections to celestial amplitudes
}

\author{
Hernán A. González, ${ }^{1}$ Andrea Puhm, ${ }^{2}$ and Francisco Rojas $\circledast^{3}$ \\ ${ }^{1}$ Facultad de Artes Liberales, Universidad Adolfo Ibáñez, \\ Diagonal Las Torres 2640, Peñalolén, Santiago, Chile \\ ${ }^{2}$ CPHT, CNRS, Ecole polytechnique, Institut Polytechnique. Paris, F-91128 Palaiseau, France \\ ${ }^{3}$ Facultad de Ingeniería y Ciencias, Universidad Adolfo Ibáñez, \\ Diagonal Las Torres 2640, Peñalolén, Santiago, Chile
}

(Received 16 October 2020; accepted 25 November 2020; published 30 December 2020)

\begin{abstract}
We study the effect of loop corrections to conformal correlators on the celestial sphere at null infinity. We first analyze finite one-loop celestial amplitudes in pure Yang-Mills theory and Einstein gravity. We then turn to our main focus: infrared divergent loop amplitudes in planar $\mathcal{N}=4$ super-Yang-Mills theory. We compute the celestial one-loop amplitude in dimensional regularization and show that it can be recast as an operator acting on the celestial tree-level amplitude. This extends to any loop order, and the resummation of all planar loops enables us to write down an expression for the all-loop celestial amplitude. Finally, we show that the exponentiated all-loop expression given by the Bern-Dixon-Smirnov (BDS) formula gets promoted on the celestial sphere to an operator acting on the tree-level conformal correlation function, thus yielding, the celestial BDS formula.
\end{abstract}

DOI: 10.1103/PhysRevD.102.126027

\section{INTRODUCTION}

Celestial amplitudes reveal conformal properties of fourdimensional scattering amplitudes of massless particles as the standard plane wave basis is replaced by a basis of boost eigenstates. This is achieved by a Mellin transform applied to each external state in the scattering amplitude, which maps plane waves labeled by the null momenta of the particles, or equivalently, their energy and a point on the 2-sphere as well as their helicity to so-called conformal primary wave functions. The latter are labeled by the conformal dimension $\Delta$ and the spin $J$ under the twodimensional global conformal group which arises from the action of the four-dimensional $S L(2, \mathbb{C})$ Lorentz group on the celestial sphere at null infinity.

Conformal wave functions have already been considered by Dirac [1], but recent years have seen a surge in interest in part due to their role for a potentially holographic description of asymptotically flat spacetimes [2-5]. This has in part been driven by the realization that the asymptotic symmetry group of Einstein gravity at null infinity should include an extension of the Bondi-Metzner-Sachs (BMS) group that enhances the global conformal group on the celestial sphere to the full Virasoro symmetry group. ${ }^{1}$

\footnotetext{
${ }^{1}$ The Virasoro symmetry may be further enhanced to $\operatorname{Diff}\left(S^{2}\right)$ see Ref. [6] for a discussion of this point. See also Ref. [7-10] for further results on symmetries of celestial amplitudes.

Published by the American Physical Society under the terms of the Creative Commons Attribution 4.0 International license. Further distribution of this work must maintain attribution to the author(s) and the published article's title, journal citation, and DOI. Funded by SCOAP ${ }^{3}$.
}

To understand the properties of a putative holographically dual celestial conformal field theory (CFT) at null infinity of asymptotically Minkowski spacetimes, a bottom-up approach has been pursued, starting with the identification of a conformal basis of wave functions in Refs. [7,11-14] and the computation of celestial amplitudes for various spins [15-19], which all take the form of two-dimensional correlation functions on the celestial sphere. Furthermore, the celestial analog of various soft theorems in quantum field theory have been obtained in Refs. [20-25], while collinear limits of scattering amplitudes have been shown in Refs. [26-30] to extract from celestial amplitudes the operator product expansion of conformal primaries in the putative celestial CFT. The formalism for a relativistic partial wave expansion for celestial four-point amplitudes was developed in Ref. [31] (see also Ref. [20]). A procedure for a celestial double copy relating celestial gravity and gauge theory amplitudes has been discussed in Ref. [32].

Most work so far has focused on celestial tree-level amplitudes, while quantum effects have largely been neglected. One may thus wonder how the conformal structure uncovered at tree level is affected once loop corrections are taken into account. The Mellin transform involves a sum over all energies that mixes the infrared and ultraviolet regimes. The main difficulty in computing celestial amplitudes at loop level is thus due to the integration over internal momentum loops which render the Mellin integrals divergent. ${ }^{2}$

\footnotetext{
${ }^{2}$ These difficulties are circumvented when considering the celestial analog of finite loop amplitudes whose tree-level counterparts vanish. Such amplitudes were considered in Ref. [33] for gluons and gravitons, and we will discuss the case of one-loop four-point amplitudes in pure Yang-Mills theory and Einstein gravity below.
} 
Nevertheless, there have been first attempts at understanding the conformal structure of flat space scattering amplitudes beyond tree level in Ref. [34], which studies celestial loop effects in massless scalar field theory with $\phi^{4}$ interaction.

Here, we would like to initiate the study of divergent loop corrections to celestial tree-level amplitudes involving gluons as external massless states. Due to gauge invariance, the external states are guaranteed to reach null infinity at any order in perturbation theory. We will focus on the case of maximal helicity violation (MHV) four-point amplitudes in planar $\mathcal{N}=4$ super-Yang-Mills theory. This has the benefit that the four-gluon amplitude is known to all orders in the loop expansion. To control infrared divergences, we employ the dimensional regularization method known as the four-dimensional helicity (FDH) scheme [35]. The momenta of the internal particles are taken to be in $D=$ $4-2 \epsilon$ dimensions, while the momenta of the external particles remain in $D=4$, as do the polarization vectors for both external and internal particles. The four-gluon MHV amplitude in planar $\mathcal{N}=4$ super-Yang-Mills can be conveniently written in factorized form in terms of the tree-level amplitude containing the helicity structure and an infrared divergent piece containing the information about the loop order.

Our main result is that this infrared divergent factor gets promoted, after the Mellin transform from the momentum basis to the conformal basis, to a differential operator. Thus, the celestial one-loop amplitude in planar $\mathcal{N}=4$ super-Yang-Mills can be recast as an operator acting on the celestial tree-level amplitude. ${ }^{3}$ This structure is shown to persist at any loop order. The effect of this celestial loop operator is to simultaneously shift the conformal dimensions of all the external particles. The operator is dressed with a loop-order dependent function of the conformal cross-ratio and diverges as the dimensional regulator is taken to zero.

Given that the Mellin transform effectively replaces the notion of energy with that of conformal scaling dimension, it is not too surprising that loop effects should manifest themselves as a change in the latter. Interestingly enough, though, celestial loop effects can be recast as an operator statement. This also resonates with the recent presentation in Ref. [32] of tree-level graviton and gluon amplitudes as operators acting on scalar amplitudes when the asymptotic states are taken to be in the conformal basis.

In momentum space, the four-gluon amplitude is known to all orders in the loop expansion. Moreover, the resummation of all loop contributions exponentiates yielding the BDS formula found by Bern et al. [37] based on an iterative relation uncovered by Anastasiou et al. [38]. ${ }^{4}$ Here, we

\footnotetext{
${ }^{3}$ Similar conclusions have been recently hinted at for QED and gravity [36].

${ }^{4}$ This was later confirmed in the strong coupling regime by Alday and Maldacena [39].
}

provide its celestial analog. At any loop order, there exists an operator acting on the celestial tree-level amplitude which results in a loop-order dependent shift in the conformal dimensions. The resummation of all celestial loop operators exponentiates, thus yielding the celestial BDS formula.

This paper is organized as follows. We begin with a review in Sec. II, where we set up the necessary notation. A convenient basis for discussing celestial amplitudes at tree and loop levels is introduced in Sec. II A. In Sec. II B, we review some of the salient features of celestial amplitudes, and we give a tree-level example in Sec. II C. We discuss loop amplitudes in Sec. III. First, we compute in Sec. III A celestial amplitudes for one-loop processes in Yang-Mills theory for which the tree-level amplitudes vanish. We then move on to the main focus of this paper in Sec. III B and analyze how divergent loop corrections in planar $\mathcal{N}=4$ super-Yang-Mills theory correct the corresponding tree-level celestial correlators. We show in Sec. III B 1 that the one-loop four-gluon celestial amplitudes can be recast as an operator acting on the celestial tree-level amplitude and generalize this result to $\ell$ loops in Sec. III B 2. The exponentiated resummation of the allloop result then gets promoted to an operator statement yielding the celestial analog of the BDS formula. In the Appendix A, we make use of the general expression for celestial amplitudes introduced in Sec. II to analyze treelevel amplitudes in massless QED in Appendix A 1 and finite one-loop amplitudes in Einstein gravity in Appendix A 2.

\section{SETUP}

In this section, we lay out a convenient basis for expressing celestial amplitudes at tree and loop levels. We consider scattering amplitudes in four dimensions ${ }^{5}$ with external massless particles. Each external particle is labeled by a momentum $p_{i}^{\mu}$, a helicity $\ell_{i}$, and a sign distinguishing incoming from outgoing states. We parametrize $p_{i}^{\mu}$ in terms of points $\left(z_{i}, \bar{z}_{i}\right)$ on the two-dimensional celestial sphere through the map

$p_{i}^{\mu}=\frac{1}{2} \omega_{i}\left(1+\left|z_{i}\right|^{2}, z_{i}+\bar{z}_{i},-i\left(z_{i}-\bar{z}_{i}\right), 1-\left|z_{i}\right|^{2}\right)$,

with $\omega_{i} \geq 0$.

\section{A. Convenient basis for four-point amplitudes}

Our focus will be on four-point amplitudes

$$
\begin{aligned}
\mathcal{A}\left(\left\{\omega_{i}, \ell_{i}, z_{i}, \bar{z}_{i}\right\}\right)= & A\left(1^{\ell_{1}}, 2^{\ell_{2}}, 3^{\ell_{3}}, 4^{\ell_{4}}\right) \\
& \times \delta\left(p_{1}+p_{2}-p_{3}-p_{4}\right) .
\end{aligned}
$$

\footnotetext{
${ }^{5}$ We work in the $(+---)$ signature.
} 
Without loss of generality, we assume that the stripped amplitude can be split as

$$
A\left(1^{\ell_{1}}, 2^{\ell_{2}}, 3^{\ell_{3}}, 4^{\ell_{4}}\right)=\mathcal{B}(s, t) \mathcal{R}_{\left(\ell_{1}, \ell_{2}, \ell_{3}, \ell_{4}\right)},
$$

where $\mathcal{B}(s, t)$ is a (not necessarily analytic) function of two of the Mandelstam variables $s=\left(p_{1}+p_{2}\right)^{2}, t=\left(p_{1}-p_{4}\right)^{2}$, and $u=\left(p_{1}-p_{3}\right)^{2}$, and the rational function $\mathcal{R}_{\left(\ell_{1}, \ell_{2}, \ell_{3}, \ell_{4}\right)}$ carries the helicity structure of the external particles. The latter is a function of the spinor products $\langle i j\rangle$ and $[i j]$ defined by ${ }^{6}$

$$
\begin{aligned}
& \langle i j\rangle=\epsilon_{\alpha \beta} \lambda_{i}^{\alpha} \lambda_{j}^{\beta}=\sqrt{\omega_{i} \omega_{j}} z_{i j}, \\
& {[i j]=-\epsilon_{\dot{\alpha} \dot{\beta}} \tilde{\lambda}_{i}^{\alpha} \tilde{\lambda}_{j}^{\dot{\beta}}=-\sqrt{\omega_{i} \omega_{j}} \bar{z}_{i j},}
\end{aligned}
$$

where $z_{i j}=z_{i}-z_{j}$ and $\bar{z}_{i j}=\bar{z}_{i}-\bar{z}_{j}$, as well as the conformally invariant cross-ratio

$$
r=\frac{z_{12} z_{34}}{z_{23} z_{41}}
$$

which is related to the four-dimensional scattering angle in the center of mass frame $\theta$ through

$$
r=-\frac{s}{t}=\csc ^{2}\left(\frac{\theta}{2}\right)
$$

It follows that $r>1$.

Since all the information about the helicities of the external states is encoded in the function $\mathcal{R}_{\left(\ell_{1}, \ell_{2}, \ell_{3}, \ell_{4}\right)}$, it must satisfy

$$
\hat{\ell}_{i} \mathcal{R}_{\left(\ell_{1}, \ell_{2}, \ell_{3}, \ell_{4}\right)}=\ell_{i} \mathcal{R}_{\left(\ell_{1}, \ell_{2}, \ell_{3}, \ell_{4}\right)},
$$

for the helicity operator for the $i$ th particle

$$
\hat{\ell}_{i}=\frac{1}{2}\left(-\lambda_{i}^{\alpha} \frac{\partial}{\partial \lambda_{i}^{\alpha}}+\tilde{\lambda}_{i}^{\dot{\alpha}} \frac{\partial}{\partial \tilde{\lambda}_{i}^{\dot{\alpha}}}\right) .
$$

We may express a solution to (2.7) in terms of powers of Lorentz invariant functions $R_{i}$, with the defining property that $\hat{\ell}_{i} R_{j}=\delta_{i j} R_{j}$, as

$$
\mathcal{R}_{\left(\ell_{1}, \ell_{2}, \ell_{3}, \ell_{4}\right)}=r^{\alpha_{1}}(r-1)^{\alpha_{2}} \prod_{i=1}^{4} R_{i}^{\ell_{i}},
$$

${ }^{6}$ The spinors $\lambda_{i}^{\alpha}$ and $\tilde{\lambda}_{i}^{\dot{\alpha}}$ can be read off from

$$
p^{\dot{\alpha} \alpha}=\bar{\sigma}_{\mu}^{\dot{\alpha} \alpha} p^{\mu}=\omega\left[\begin{array}{cc}
1 & z \\
\bar{z} & |z|^{2}
\end{array}\right]=\underbrace{\sqrt{\omega}\left[\begin{array}{l}
1 \\
\bar{z}
\end{array}\right]}_{\tilde{\lambda}^{\alpha}} \underbrace{\sqrt{\omega}\left[\begin{array}{ll}
1 & z
\end{array}\right]}_{\lambda^{\alpha}} .
$$

with $\alpha_{1}$ and $\alpha_{2}$ real numbers. A basis of functions $R_{i}$ that depend on the energies $\omega_{i}$ of the external particles was given in Ref. [41]. For our purposes, it is more convenient to express $\mathcal{R}$ in terms of functions that depend exclusively on the differences of points $\left(z_{i j}, \bar{z}_{i j}\right)$ on the celestial sphere. Such a set of $R_{i}$ is given by

$$
\begin{array}{rlrl}
R_{1} & =\left(\frac{[12][13]\langle 23\rangle}{\langle 12\rangle\langle 13\rangle[32]}\right)^{\frac{1}{2}}, & R_{2} & =\left(\frac{[12][23]\langle 13\rangle}{\langle 12\rangle\langle 23\rangle[31]}\right)^{\frac{1}{2}}, \\
R_{3} & =\left(\frac{[13][23]\langle 12\rangle}{\langle 13\rangle\langle 23\rangle[21]}\right)^{\frac{1}{2}}, & R_{4}=\frac{[24]}{\langle 24\rangle}\left(\frac{[31]\langle 12\rangle\langle 23\rangle}{\langle 13\rangle[12][23]}\right)^{\frac{1}{2}} .
\end{array}
$$

The four-point amplitude (2.3) can then be expressed as

$$
\begin{aligned}
A\left(1^{\ell_{1}}, 2^{\ell_{2}}, 3^{\ell_{3}}, 4^{\ell_{4}}\right) & =r^{\alpha_{1}}(r-1)^{\alpha_{2}} \mathcal{B}(s, t)\left(\frac{z_{12}}{\bar{z}_{12}}\right)^{-\frac{1}{2}\left(\ell_{1}+\ell_{2}-\ell_{3}-\ell_{4}\right)} \\
& \times\left(\frac{z_{13}}{\bar{z}_{13}}\right)^{-\frac{1}{2}\left(\ell_{1}-\ell_{2}+\ell_{3}+\ell_{4}\right)}\left(\frac{z_{23}}{\bar{z}_{23}}\right)^{\frac{1}{2}\left(\ell_{1}-\ell_{2}-\ell_{3}+\ell_{4}\right)} \\
& \times\left(\frac{z_{24}}{\bar{z}_{24}}\right)^{-\ell_{4}} .
\end{aligned}
$$

Notice that no mention to perturbation theory has been made, and as long as the amplitude is of the form (2.11), the statements made so far apply to the exact four-point $\mathcal{S}$-matrix element.

\section{B. Celestial amplitudes}

Celestial amplitudes are obtained by performing a Mellin transform on each of the $n$ external particles in a scattering process,

$$
\begin{aligned}
& \tilde{\mathcal{A}}\left(\left\{\Delta_{i}, J_{i}, z_{i}, \bar{z}_{i}\right\}\right) \\
& \quad=\prod_{k=1}^{n}\left(\int_{0}^{\infty} d \omega_{k} \omega_{k}^{\Delta_{k}-1}\right) \mathcal{A}\left(\left\{\omega_{i}, \ell_{i}, z_{i}, \bar{z}_{i}\right\}\right) .
\end{aligned}
$$

Under $S L(2, \mathbb{C})$ Lorentz transformations, they have been shown to transform as $[11,16,19]$

$$
\begin{aligned}
\tilde{\mathcal{A}} & \left(\left\{\Delta_{j}, J_{i} ; \frac{a z_{i}+b}{c z_{i}+d}, \frac{\bar{a} \bar{z}_{i}+\bar{b}}{\bar{c} \bar{z}_{i}+\bar{d}}\right\}\right) \\
& =\prod_{j=1}^{n}\left(\left(c z_{j}+d\right)^{\Delta_{j}+J_{j}}\left(\bar{c} \bar{z}_{j}+\bar{d}\right)^{\Delta_{j}-J_{j}}\right) \tilde{\mathcal{A}}\left(\left\{\Delta_{i}, J_{i} ; z_{i}, \bar{z}_{i}\right\}\right),
\end{aligned}
$$

where $\Delta_{j}$ are the conformal dimensions and $J_{j} \equiv \ell_{j}$ are the spins of operators inserted at the points $\left(z_{j}, \bar{z}_{j}\right) \in S^{2}$. Celestial amplitudes thus share conformal properties with 
correlation functions on the celestial sphere. Indeed, evaluating (2.12) for the four-point amplitude (2.2) using

$$
\begin{aligned}
\delta^{(4)} & \left(p_{1}+p_{2}-p_{3}-p_{4}\right) \\
= & \frac{4}{\omega_{4}\left|z_{14}\right|^{2}\left|z_{23}\right|^{2}} \delta(r-\bar{r}) \delta\left(\omega_{1}-\frac{z_{24} \bar{z}_{34}}{z_{12} \bar{z}_{13}} \omega_{4}\right) \\
& \times \delta\left(\omega_{2}+\frac{z_{14} \bar{z}_{34}}{z_{12} \bar{z}_{23}} \omega_{4}\right) \delta\left(\omega_{3}+\frac{z_{24} \bar{z}_{14}}{z_{23} \bar{z}_{13}} \omega_{4}\right)
\end{aligned}
$$

yields the celestial four-point amplitude (up to a numerical factor)

$$
\tilde{\mathcal{A}}\left(\left\{\Delta_{i}, J_{i}, z_{i}, \bar{z}_{i}\right\}\right)=f(r, \bar{r}) \prod_{i<j}^{4} z_{i j}^{\frac{h}{3}-h_{i}-h_{j}} \bar{z}_{i j}^{\frac{\bar{h}}{3}-\bar{h}_{i}-\bar{h}_{j}} .
$$

The conformally invariant expression $f(r, \bar{r})$ is given by

$$
\begin{aligned}
f(r, \bar{r})= & 2 \delta(r-\bar{r}) \Theta(r-1) r^{\alpha_{1}+\frac{\Delta}{6}}(r-1)^{\alpha_{2}+\frac{\Delta}{6}} \\
& \times \int_{0}^{\infty} d w w^{\frac{\Delta-6}{2}} \mathcal{B}(r w,-w),
\end{aligned}
$$

with $\Delta \equiv \sum_{i=1}^{4} \Delta_{i}$. The conformal weights of the external wave functions introduced in (2.15) are given by

$$
h_{k}=\frac{1}{2}\left(\Delta_{k}+J_{k}\right), \quad \bar{h}_{k}=\frac{1}{2}\left(\Delta_{k}-J_{k}\right),
$$

where the two-dimensional spins $J_{k}$ are identified with the helicities $\ell_{k}$ of the four-dimensional particles crossing null infinity and the conformal dimensions $\Delta_{k}$ of finite energy wave functions are restricted to lie on the principal continuous series of the $S L(2, \mathbb{C})$ Lorentz group[11], namely

$$
\Delta_{k}=1+i \lambda_{k} \quad \text { with } \quad \lambda_{k} \in \mathbb{R}, \quad J_{k} \equiv \ell_{k} .
$$

\section{Example: Celestial gluons at tree level}

We illustrate the above prescription for celestial fourgluon amplitudes at tree level in pure Yang-Mills theory which were first discussed in Refs. [16,19]. The MHV four-gluon amplitude is given by (2.2) with the stripped amplitude

$A_{\text {tree }}\left(1^{-}, 2^{-}, 3^{+}, 4^{+}\right)=g^{2} \frac{\langle 12\rangle^{3}}{\langle 23\rangle\langle 34\rangle\langle 41\rangle}=g^{2} r \frac{z_{12} \bar{z}_{34}}{\bar{z}_{12} z_{34}}$,

where in the last step we used the constraint (2.14). Comparing with (2.11) for $\left(\ell_{1}, \ell_{2}, \ell_{3}, \ell_{4}\right)=(-1,-1,1,1)$, we read off $\alpha_{1}=1, \alpha_{2}=0$, and the function $\mathcal{B}(s, t)=g^{2}$ just corresponds to the coupling. ${ }^{7}$ The corresponding celestial amplitude is given by the two-dimensional fourpoint correlation function (2.15) of gluons with conformal weights $\left(h_{k}, \bar{h}_{k}\right)=\left(\frac{i}{2} \lambda_{k}, 1+\frac{i}{2} \lambda_{k}\right)$ for negative helicity and $\left(h_{k}, \bar{h}_{k}\right)=\left(1+\frac{i}{2} \lambda_{k}, \frac{i}{2} \lambda_{k}\right)$ for positive helicity. The conformally invariant expression $f(r, \bar{r})$ is given by

$f_{\text {tree }}(r, \bar{r})=2 g^{2} \delta(r-\bar{r}) \Theta(r-1) r^{1+\frac{\Delta}{6}}(r-1)^{\frac{\Delta}{6}} \mathcal{I}(\lambda)$,

where

$$
\mathcal{I}(\lambda) \equiv \int_{0}^{\infty} \frac{d w}{w} w^{i \frac{\lambda}{2}}=4 \pi \delta(\lambda),
$$

with $\lambda=\sum_{k=1}^{4} \lambda_{k}$. Enforcing (2.21) sets $\Delta=4$ and thus yields

$f_{\text {tree }}(r, \bar{r})=8 \pi g^{2} \delta(r-\bar{r}) \Theta(r-1) r^{\frac{5}{3}}(r-1)^{\frac{2}{3}} \delta(\lambda)$.

Because the integral in (2.16) is marginally convergent for celestial tree-level Yang-Mills amplitudes, it can be interpreted as a distribution given by (2.21). This is no longer true for celestial amplitudes at loop level, which we will discuss in the next section. Before doing so, let us comment that, while we have focused here on tree-level amplitudes in pure Yang-Mills theory in order to set the stage for the discussion of celestial gluon amplitudes at loop level, the prescription discussed in Secs. II A-II B is more broadly applicable. In Appendix A, we analyze tree-level amplitudes in massless QED theory and finite one-loop Einstein gravity processes.

\section{CELESTIAL LOOP AMPLITUDES}

In this section, we discuss how loop corrections modify celestial amplitudes. We first consider in Sec. III A processes in pure Yang-Mills theory that vanish at tree level and yield finite one-loop amplitudes. In Sec. III B, we move on to the more interesting case of MHV amplitudes in planar $\mathcal{N}=4$ super-Yang-Mills theory. We compute the one-loop celestial amplitude in Sec. III B 1 and extend this result to all loops in Sec. III B 2.

\section{A. Finite one-loop amplitudes in Yang-Mills}

We consider loop corrected amplitudes for theories involving only external gluons. The simplest processes are the ones that vanish at tree level. ${ }^{8}$ This is the case for gluon amplitudes whose helicities are ++++ and -+++ as well as the opposite helicity cases. For the

\footnotetext{
${ }^{7}$ Here and henceforth, we omit a factor $i(2 \pi)^{4}$.

${ }^{8}$ Recently, we learned about the work of Albayrak et al. [33], which has some overlap with this subsection and with Appendix A 2.
} 
all helicity plus and all helicity minus amplitudes, we have [42-44]

$A\left(1^{ \pm}, 2^{ \pm}, 3^{ \pm}, 4^{ \pm}\right)=g^{4}\left(\frac{[12][34]}{\langle 12\rangle\langle 34\rangle}\right)^{ \pm 1}=g^{4}\left(\frac{\bar{z}_{12} \bar{z}_{34}}{z_{12} z_{34}}\right)^{ \pm 1}$,

while the mixed helicity amplitudes are given by

$A\left(1^{-}, 2^{+}, 3^{+}, 4^{+}\right)=g^{4} \frac{\langle 13\rangle[31][24]^{2}}{[12]\langle 23\rangle\langle 34\rangle[41]}=g^{4} \frac{z_{13} \bar{z}_{31} \bar{z}_{24}^{2}}{\bar{z}_{12} z_{23} \bar{z}_{34} \bar{z}_{41}}$,

with the opposite helicity amplitude obtained by exchanging $z_{i j} \leftrightarrow \bar{z}_{i j}$. In pure Yang-Mills theory, these amplitudes correspond to the single trace and color ordered contribution to four-gluon processes. They also represent the leading contribution to photon-photon or gluon-gluon scattering from the box diagram of massless QED or QCD with a fermion running inside the loop [45-47].

Because the amplitudes (3.1) and (3.2) are again of the form (2.3), the conformal weights are directly obtained from (2.17). The new information from these loop processes in the bulk lies in the computation of the conformally invariant factor (2.16), which yields

$$
\begin{aligned}
f_{1-\text { loop }}(r, \bar{r})= & 8 \pi g^{4} \delta(\lambda) \delta(r-\bar{r}) \Theta(r-1)(r-1)^{\frac{2}{3}} \\
& \times \begin{cases}r^{\frac{2}{3}}, & ( \pm, \pm, \pm, \pm) \\
r^{\frac{5}{3}}, & (\mp, \pm, \pm, \pm)\end{cases}
\end{aligned}
$$

Notice that here we were again able to make use of (2.21), reflecting the fact that the above amplitudes are finite even at one loop. For infrared divergent amplitudes which we will discuss in the following, the distribution involving the sum of the conformal dimensions will be promoted to an operator statement.

\section{B. Infrared divergent amplitudes in planar $\mathcal{N}=4$ super-Yang-Mills}

We now turn to the more interesting case of MHV amplitudes and how loop corrections modify the celestial tree-level correlators involving gluons as external states. Because these amplitudes do not vanish at tree level, their loop corrections (in momentum space) typically suffer from both UV and IR divergences. While the former may be taken care of by renormalization, the latter are more subtle. ${ }^{9}$ Here, to control infrared divergences, we employ the dimensional regularization method known as the FDH scheme [35]. The momenta of the internal particles are in $D=4-2 \epsilon$ dimensions, whereas all polarization vectors (internal and external) and the momenta of

\footnotetext{
${ }^{9}$ For a recent discussion of IR divergences, see Refs. [48,49].
}

the external states remain in $D=4$. We focus on planar amplitudes in $\mathcal{N}=4$ super-Yang-Mills theory. Besides being UV finite, this has the benefit that the four-gluon amplitude is known to all orders in the loop expansion (in the 't Hooft coupling). The MHV four-gluon amplitude can be conveniently written as ${ }^{10}$

$$
A_{\text {all loops }}=M_{\epsilon} A_{\text {tree }}
$$

where the scalar factor

$$
M_{\epsilon}=1+\sum_{\ell=1}^{\infty} a^{\ell} M_{\epsilon}^{(\ell)}
$$

depends on the Mandelstam invariants $s$ and $t$ as well as the dimensional regulator. Here, $a \equiv \frac{g^{2} N}{8 \pi^{2}}\left(4 \pi e^{-\gamma_{E}}\right)^{\epsilon}$ is the 't Hooft coupling, and $\gamma_{E}$ is the Euler-Mascheroni constant. The tree-level amplitude $A_{\text {tree }}$ is the same as in pure YangMills, which for the choice of helicities $\left(1^{-}, 2^{-}, 3^{+}, 4^{+}\right)$is given in (2.19). As shown in Ref. [37], the amplitude (3.5) exponentiates according to the BDS formula

$$
M_{\epsilon}=\exp \left(\sum_{\ell=1}^{\infty} a^{\ell}\left(f_{\epsilon}^{(\ell)} M_{\ell \epsilon}^{(1)}+C^{(\ell)}+E_{\epsilon}^{(\ell)}\right)\right),
$$

where $f_{\epsilon}^{(\ell)}$ are regular functions of $\epsilon$ and are directly related to the cusp and collinear anomalous dimensions, $C^{(\ell)}$ are numerical constants, and $E_{\epsilon}^{(\ell)}$ is of order $\mathcal{O}(\epsilon)$. In the following, we will discuss the celestial analog of the above statements.

\section{Celestial gluons at one loop}

The one-loop contribution [51]

$$
A_{1-\text { loop }}=a M_{\epsilon}^{(1)} A_{\text {tree }}
$$

is obtained by solving the scalar box integral

$$
\begin{aligned}
M_{\epsilon}^{(1)}= & -\frac{1}{2}\left(\mu^{2} e^{\gamma_{E}}\right)^{\epsilon} \int \frac{d^{D} p}{i \pi^{D / 2}} \\
& \times \frac{s t}{p^{2}\left(p+p_{1}\right)^{2}\left(p+p_{1}+p_{2}\right)^{2}\left(p+p_{4}\right)^{2}},
\end{aligned}
$$

where $\mu$ is the dimensional regularization scale. This integral can be explicitly evaluated in terms of hypergeometric functions (see, for instance, Appendix E of Ref. [52])

\footnotetext{
${ }^{10}$ See, for instance, Sec. IV 3 in Ref. [50].
} 


$$
\begin{aligned}
M_{\epsilon}^{(1)}= & -\frac{1}{\epsilon^{2}} e^{\epsilon \gamma_{E}} \gamma_{\Gamma} \frac{t}{\mu^{2}}\left[\left(\frac{\mu^{2}}{-t}\right){ }^{1+\epsilon}{ }_{2} F_{1}\left(-\epsilon, 1 ; 1-\epsilon ; 1+\frac{t}{s}\right)\right. \\
& \left.-\left(\frac{\mu^{2}}{-s}\right)^{1+\epsilon}{ }_{2} F_{1}\left(1,1 ; 1-\epsilon ; 1+\frac{t}{s}\right)\right]
\end{aligned}
$$

where $\gamma_{\Gamma}=\Gamma(1+\epsilon) \Gamma^{2}(1-\epsilon) / \Gamma(1-2 \epsilon)$. Note that we can express (3.9) as

$$
M_{\epsilon}^{(1)}=\left(\frac{\mu^{2}}{-t}\right)^{\epsilon} \mathcal{F}_{1}(r, \epsilon),
$$

where $\mathcal{F}_{1}(r, \epsilon)$ depends on the Mandelstam invariants $s$ and $t$ only through the conformally invariant cross-ratio $r=-s / t$ introduced in (2.6) and has double poles in $\epsilon$ inherited from the dimensionally regularized one-loop integral whose explicit form can be read off from (3.9).

In computing the celestial four-gluon amplitude (2.15) at one loop in planar $\mathcal{N}=4$ super-Yang-Mills, we thus see that the main difference to the tree-level result lies in the (now divergent) Mellin integral in (2.16) where $w=-t$, namely

$$
\int_{0}^{\infty} \frac{d w}{w} w^{i \frac{\lambda}{2}} \mathcal{B}(r w,-w)=a \mu^{2 \epsilon} \mathcal{F}_{1}(r, \epsilon) \int_{0}^{\infty} \frac{d w}{w} w^{i \frac{\lambda}{2}-\epsilon}
$$

The one-loop contribution to the conformally invariant factor is given by

$$
\begin{aligned}
f_{1-\text { loop }}(r, \bar{r}, \epsilon)= & 2 a g^{2} \mu^{2 \epsilon} \mathcal{F}_{1}(r, \epsilon) \delta(r-\bar{r}) \Theta(r-1) \\
& \times r^{1+\frac{\Delta}{6}}(r-1)^{\frac{\Delta}{6}} \mathcal{I}(\lambda+2 i \epsilon) .
\end{aligned}
$$

While this result shares some similarities with $f_{\text {tree }}$ given in (2.20), it notably differs from the tree-level result of the Mellin integral (2.21) by a shift in the argument. Interestingly, this shift can be reexpressed through the action of the differential operator

$$
\mathcal{I}(\lambda+2 i \epsilon)=e^{2 i \epsilon \partial_{\lambda}} \mathcal{I}(\lambda)
$$

This suggests that celestial amplitudes at loop level may be obtainable from tree-level ones through the action of appropriate differential operators. Indeed, we find that the celestial one-loop amplitude can be written as

$$
\tilde{\mathcal{A}}_{1-\text { loop }}=a \hat{\mathcal{M}}_{\epsilon}^{(1)} \tilde{\mathcal{A}}_{\text {tree }}
$$

for the celestial one-loop operator

$$
\hat{\mathcal{M}}_{\epsilon}^{(1)}=\mathcal{F}_{1}(r, \epsilon) \hat{\mathcal{P}}^{\epsilon}
$$

where we defined
$\hat{\mathcal{P}}=\mu^{2} r^{\frac{1}{3}}(r-1)^{\frac{1}{3}} \prod_{i<j}\left(z_{i j} \bar{z}_{i j}\right)^{-\frac{1}{6}} \exp \left(\frac{i}{2} \sum_{k=1}^{4} \frac{\partial}{\partial \lambda_{k}}\right)$.

Notice that $\hat{\mathcal{P}}$ is related to the operator $P_{+, k}=e^{\frac{1}{2}\left(\partial_{h_{k}}+\partial_{\bar{h}_{k}}\right)}$ introduced in Ref. [9] whose effect is to shift the conformal dimension $\Delta_{k} \rightarrow \Delta_{k}+1$ of the individual gluons. There, the sum over all $P_{+, k}$ was shown to annihilate celestial amplitudes as expected by translation invariance. Here, $\hat{\mathcal{P}}$ involves instead the product over all $P_{+, k}$. Moreover, the prefactor in (3.16) can be recast as a correlation function of four scalar primaries with conformal weights $h_{i}=\bar{h}_{i}=\frac{1}{4}$. Its appearance is not surprising, as the action of the exponential operator shifts all the conformal weights by the same amount $h_{i} \rightarrow h_{i}-\frac{\epsilon}{4}$ and hence the role of the prefactor in (3.16) is precisely to cancel these extra factors.

Thus, we see that the celestial one-loop amplitude in planar $\mathcal{N}=4$ super-Yang Mills can be recast as an infrared divergent operator acting on the celestial tree-level amplitude. The one-loop factor $M_{\epsilon}^{(1)}$ multiplying the tree-level amplitude in (3.7) gets promoted to the celestial one-loop operator $\hat{\mathcal{M}}_{\epsilon}^{(1)}$ acting on the celestial tree-level amplitude (3.14). In the following, we generalize this statement to all loops.

\section{Celestial gluons at all loops}

The $\ell$-loop contribution to the four-gluon planar amplitude is

$$
A_{\ell \text {-loop }}=a^{\ell} M_{\epsilon}^{(\ell)} A_{\text {tree }}
$$

where $M_{\epsilon}^{(\ell)}$ can be written as $[53,54]$

$$
M_{\epsilon}^{(\ell)}=\left(\frac{\mu^{4}}{s t}\right)^{\frac{\ell_{\epsilon}}{2}} \mathcal{G}_{\ell}(r, \epsilon) .
$$

For our purposes, using $s=-t / r$, it is more convenient to write this instead as

$$
M_{\epsilon}^{(\ell)}=\left(\frac{\mu^{2}}{-t}\right)^{\ell \epsilon} \mathcal{F}_{\ell}(r, \epsilon)
$$

where $\mathcal{F}_{\ell}=(-r)^{-\frac{\ell \epsilon}{2}} \mathcal{G}_{\ell}$ has poles starting at $\epsilon^{-2 \ell}$. Explicit expression for $\mathcal{F}_{\ell}$ to all orders in $\epsilon$ can be obtained in terms of Mellin-Barnes integral representations for $\ell=2$ in Ref. [55], for $\ell=3$ in Ref. [37], and for $\ell=4$ in Ref. [56]. Notice that, as in the one-loop case, the dependence of the $\ell$-loop amplitude on the Mandelstam variables is a simple power of $t$ factored out in (3.19), while $\mathcal{F}_{\ell}(\epsilon, r)$ depends only on the conformal cross-ratio, thus making the Mellin integral in (2.16) straightforward to compute. The celestial one-loop expression (3.14) generalizes to $\ell$ loops as 


$$
\tilde{\mathcal{A}}_{\ell \text {-loop }}=a^{\ell} \hat{\mathcal{M}}_{\epsilon}^{(\ell)} \tilde{\mathcal{A}}_{\text {tree }}
$$

with the celestial $\ell$-loop operator given by

$$
\hat{\mathcal{M}}_{\epsilon}^{(\ell)}=\mathcal{F}_{\ell}(r, \epsilon) \hat{\mathcal{P}}^{\ell \epsilon}
$$

We can immediately resum the perturbative expansion of the four-gluon celestial amplitude to all loop orders, yielding

$$
\tilde{\mathcal{A}}_{\text {all loops }}=\hat{\mathcal{M}}_{\epsilon} \tilde{\mathcal{A}}_{\text {tree }},
$$

with

$$
\hat{\mathcal{M}}_{\epsilon}=1+\sum_{\ell=1}^{\infty} a^{\ell} \hat{\mathcal{M}}_{\epsilon}^{(\ell)}
$$

Inspired by the BDS exponentiation [37], we now show that the infinite sum (3.23) can be recast as an exponential operator acting on the celestial tree-level amplitude. To do so, we make use of the identity

$$
1+\sum_{\ell=1}^{\infty} a^{\ell} \hat{\mathcal{M}}_{\epsilon}^{(\ell)}=\exp \left(\sum_{L=1}^{\infty} a^{L}\left(\hat{\mathcal{M}}_{\epsilon}^{(L)}-\hat{X}^{(L)}\left[\hat{\mathcal{M}}_{\epsilon}^{(\ell)}\right]\right)\right)
$$

where we introduced

$$
\hat{X}^{(L)}\left[\hat{\mathcal{M}}_{\epsilon}^{(\ell)}\right]=\hat{\mathcal{M}}_{\epsilon}^{(L)}-\left.\log \left(1+\sum_{\ell=1}^{\infty} a^{\ell} \hat{\mathcal{M}}_{\epsilon}^{(\ell)}\right)\right|_{a^{L} \text {-term }},
$$

which only depends on the lower-loop operator $\hat{\mathcal{M}}_{\epsilon}^{(\ell)}$ with $\ell<L$. We obtain

$\hat{\mathcal{M}}_{\epsilon}=\exp \left(\sum_{L=1}^{\infty} a^{L}\left(\mathcal{F}_{L}(r, \epsilon)-X^{(L)}\left[\mathcal{F}_{\ell}(r, \epsilon)\right]\right) \hat{\mathcal{P}}^{L \epsilon}\right)$.

Note that $X^{(L)}$ in the above expression is no longer an operator, as opposed to $\hat{X}^{(L)}$ in (3.24). At this point, we can make use of the explicit form of $\mathcal{F}_{\ell}$ known from the BDS formula [37]

$$
\begin{aligned}
\mathcal{F}_{L}(r, \epsilon)= & X^{(L)}\left[\mathcal{F}_{\ell}(r, \epsilon)\right]+f_{\epsilon}^{(L)} \mathcal{F}_{1}(r, L \epsilon) \\
& +C^{(L)}+\mathcal{E}_{L}(r, \epsilon),
\end{aligned}
$$

where $\mathcal{E}_{L}$ are noniterating $\mathcal{O}(\epsilon)$ contributions. With this, we find the celestial BDS formula

$$
\begin{aligned}
\tilde{\mathcal{A}}_{\text {all loops }}= & \exp \left(\sum _ { L = 1 } ^ { \infty } a ^ { L } \left(f_{\epsilon}^{(L)} \mathcal{F}_{1}(r, L \epsilon)+C^{(L)}\right.\right. \\
& \left.\left.+\mathcal{E}_{L}(r, \epsilon)\right) \hat{\mathcal{P}}^{L \epsilon}\right) \tilde{\mathcal{A}}_{\text {tree }}
\end{aligned}
$$

This demonstrates, for the case of MHV amplitudes in planar $\mathcal{N}=4$ super-Yang-Mills that celestial amplitudes at loop level can be obtained through the action of an exponential operator on the celestial tree-level amplitudes.

\section{CONCLUSIONS}

We have analyzed the role played by loop corrections on celestial correlators. We first showed that for finite loop amplitudes, celestial amplitudes can be defined unambiguously. Then, we have considered infrared divergent amplitudes in $\mathcal{N}=4$ superYang-Mills, where we have shown that planar celestial four-point gluon amplitudes can be defined as a differential operator (containing the infrared divergences) acting on the tree level correlator. At $l$-loop this expression is given by (3.14) and at all loop orders we have obtained expression (3.28).

While we have focused here on the four-gluon amplitude, one could analyze higher-point amplitudes at loop level in $\mathcal{N}=4$ super-Yang-Mills as well as other theories with less symmetry. A preliminary study shows that a similar operator structure as the one we uncovered here seems to arise for pure Yang-Mills theory [40]. It would be interesting to investigate whether this pattern persists in other theories. In Ref. [19], the authors computed celestial tree-level amplitudes of four massless states in the open sector of the type I string. A natural next step is to compute the celestial one-loop string amplitude and see if it can be recast as an operator acting on the celestial tree-level string amplitude. If that is the case, and since conformal invariance on the worldsheet intimately ties infrared divergences to ultraviolet ones through the open/closed string duality, it would be appealing to explore how this duality manifests itself on the celestial sphere. We leave these interesting questions for the future.

\section{ACKNOWLEDGMENTS}

We would like to thank Fernando Alday, Guillaume Bossard, Eduardo Casali, Gastón Giribet, Andrés Gomberoff, Georgios Papathanasiou, Anders Schreiber, and William Torres-Bobadilla for discussions and comments on the draft. The work of H. G. has been supported by FONDECYT Grant No. 11190427. The work of F. R. has been supported by FONDECYT Grant No. 11171148. A.P. was supported in part by the European Research Council (ERC) under the EuropeanUnions Horizon 2020 research and innovation programme(Grant Agreement No 852386) and would like to thank the Facultad de 
Ingeniería y Ciencias at Universidad Adolfo Ibáñez for hospitality during her visit.

\section{APPENDIX A: MORE CELESTIAL AMPLITUDES}

In this Appendix, we make use of (2.11) to analyze treelevel amplitudes in massless QED and finite one-loop Einstein gravity processes.

\section{Celestial tree-level massless QED}

The asymptotic states of massless QED are massless electrons, positrons, and photons. We first analyze processes involving only fermions in the external states. The nonvanishing amplitudes correspond to two fermions of positive helicities and two fermions of negative helicities. These are given by (see, e.g., Ref. [57])

$$
\begin{aligned}
& A\left(1^{\frac{1}{2}}, 2^{-\frac{1}{2}}, 3^{-\frac{1}{2}}, 4^{\frac{1}{2}}\right)=-2 e^{2} \frac{[14]\langle 23\rangle}{u}=\frac{2 e^{2}}{r-1}\left(\frac{z_{23} \bar{z}_{14}}{\bar{z}_{23} z_{14}}\right)^{\frac{1}{2}}, \\
& A\left(1^{\frac{1}{2}}, 2^{-\frac{1}{2}}, 3^{\frac{1}{2}}, 4^{-\frac{1}{2}}\right)=-2 e^{2} \frac{[13]\langle 24\rangle}{t}=\frac{2 e^{2}}{r-1}\left(\frac{z_{24} \bar{z}_{13}}{\bar{z}_{24} z_{13}}\right)^{\frac{1}{2}}, \\
& A\left(1^{\frac{1}{2}}, 2^{\frac{1}{2}}, 3^{-\frac{1}{2}}, 4^{-\frac{1}{2}}\right)=-2 e^{2}[12]\langle 34\rangle \frac{s}{t u}=\frac{2 e^{2} r^{2}}{r-1}\left(\frac{z_{34} \bar{z}_{12}}{\bar{z}_{34} z_{12}}\right)^{\frac{1}{2}},
\end{aligned}
$$

with the remaining cases given by the complex conjugation. Comparing these expressions with (2.11) yields $\mathcal{B}(s, t)=2 e^{2}$ for all massless QED amplitudes with external fermions. The conformally invariant expressions for the amplitudes in (A1) are given by

$$
\begin{aligned}
f_{\frac{1}{2}}(r, \bar{r})= & 8 \pi e^{2} \delta(r-\bar{r}) \Theta(r-1) \delta(\lambda) \\
& \times \begin{cases}r^{\frac{2}{3}}(r-1)^{\frac{2}{3}}, & \left( \pm \frac{1}{2}, \mp \frac{1}{2}, \mp \frac{1}{2}, \pm \frac{1}{2}\right) \\
r^{\frac{2}{3}}(r-1)^{-\frac{1}{3}}, & \left( \pm \frac{1}{2}, \mp \frac{1}{2}, \pm \frac{1}{2}, \mp \frac{1}{2}\right) . \\
r^{\frac{8}{3}}(r-1)^{\frac{2}{3}}, & \left( \pm \frac{1}{2}, \pm \frac{1}{2}, \mp \frac{1}{2}, \mp \frac{1}{2}\right)\end{cases}
\end{aligned}
$$

Next, we consider amplitudes combining two external photons and two external fermions,

$$
\begin{aligned}
A\left(1^{\frac{1}{2}}, 2^{-\frac{1}{2}}, 3^{+}, 4^{-}\right) & =2 e^{2} \frac{\langle 24\rangle^{2}}{\langle 13\rangle\langle 23\rangle} \\
& =2 e^{2}(r-1)^{\frac{1}{2}} \frac{z_{24}}{\bar{z}_{24}}\left(\frac{\bar{z}_{23} \bar{z}_{13}}{z_{23} z_{13}}\right)^{\frac{1}{2}}, \\
A\left(1^{\frac{1}{2}}, 2^{-\frac{1}{2}}, 3^{-}, 4^{+}\right) & =2 e^{2} \frac{\langle 23\rangle^{2}}{\langle 14\rangle\langle 24\rangle} \\
& =2 e^{2}(r-1)^{-\frac{1}{2}} \frac{z_{23}}{\bar{z}_{23}}\left(\frac{\bar{z}_{24} \bar{z}_{14}}{z_{24} z_{14}}\right)^{\frac{1}{2}} .
\end{aligned}
$$

Again, we have $\mathcal{B}(s, t)=2 e^{2}$, and the conformally invariant expression are

$$
\begin{aligned}
f_{\left(1, \frac{1}{2}\right)}(r, \bar{r})= & 8 \pi e^{2} \delta(r-\bar{r}) \Theta(r-1) \delta(\lambda) r^{\frac{2}{3}} \\
& \times\left\{\begin{array}{ll}
(r-1)^{\frac{5}{3}}, & \left(\frac{1}{2},-\frac{1}{2},+,-\right) \\
(r-1)^{-\frac{1}{3}}, & \left(\frac{1}{2},-\frac{1}{2},-,+\right)
\end{array} .\right.
\end{aligned}
$$

Hence, celestial amplitudes in massless QED are correlators of two-dimensional conformal primaries with spin 1 and $\operatorname{spin} \frac{1}{2} \cdot{ }^{11}$

\section{Celestial pure gravity at one loop}

Finite amplitudes in pure gravity at one loop are $[59,60]$ given by

$$
\begin{aligned}
& A\left(1^{++}, 2^{++}, 3^{++}, 4^{++}\right) \\
& \quad=\kappa^{4} \frac{s^{2}-s t+t^{2}}{960} A\left(1^{+}, 2^{+}, 3^{+}, 4^{+}\right)^{2}, \\
& A\left(1^{--}, 2^{++}, 3^{++}, 4^{++}\right) \\
& \quad=\kappa^{4} \frac{s^{2}-s t+t^{2}}{2880}\left(\frac{s t}{u^{2}}\right)^{2} A\left(1^{-}, 2^{+}, 3^{+}, 4^{+}\right)^{2},
\end{aligned}
$$

where $\kappa=\sqrt{32 \pi G_{N}}$ and we have used the double copy when writing these amplitudes in terms of the fourgluon stripped amplitudes $A\left(1^{ \pm}, 2^{+}, 3^{+}, 4^{+}\right)$given in (3.1) and (3.2). For the corresponding celestial amplitudes, we find

$$
\begin{aligned}
f_{1-\text { loop }}(r, \bar{r})= & \frac{\kappa^{4}}{240} \mathcal{I}(\lambda-2 i) \delta(r-\bar{r}) \Theta(r-1) \\
& \times \begin{cases}r^{2}+r+1, & (++,++,++,++) \\
\frac{r^{2}+r+1}{3(r-1)^{2}}, & (--,++,++,++)\end{cases}
\end{aligned}
$$

with the Mellin integral $\mathcal{I}(\lambda)$ defined in (2.21). Note that this is the same type of divergence observed in celestial tree-level graviton amplitudes [19]. ${ }^{12}$ In string theory, these are rendered finite due to the exponentially damped behavior of the amplitudes at high energies [19]. Since the soft UV behavior holds perturbatively to all orders in the string loop expansion for both closed [61,62] and open strings [63]), one would expect convergence of the Mellin integrals of the corresponding celestial string amplitudes. In the $r \rightarrow \infty$ limit, one should then recover the above celestial one-loop gravity amplitude.

\footnotetext{
${ }^{11}$ Spin $\frac{1}{2}$ conformal wave packets were recently discussed in Ref. [58] to compute celestial amplitudes in $\mathcal{N}=1$ super-YangMills theory.

${ }^{12} \mathrm{~A}$ regularization of classical celestial graviton amplitudes is given by an imaginary shift of $\lambda$ corresponding to the insertion of gravitons of integer conformal dimension such as the stress tensor or its shadow [23].
} 
[1] P. A. Dirac, Wave equations in conformal space, Ann. Math. 37, 429 (1936).

[2] J. de Boer and S. N. Solodukhin, A holographic reduction of Minkowski space-time, Nucl. Phys. B665, 545 (2003).

[3] G. Barnich and C. Troessaert, Symmetries of Asymptotically Flat 4 Dimensional Spacetimes at Null Infinity Revisited, Phys. Rev. Lett. 105, 111103 (2010).

[4] G. Barnich and C. Troessaert, Aspects of the BMS/CFT correspondence, J. High Energy Phys. 05 (2010) 062.

[5] A. Strominger, Lectures on the infrared structure of gravity and gauge theory, arXiv:1703.05448.

[6] L. Donnay, S. Pasterski, and A. Puhm, Asymptotic symmetries and celestial CFT, J. High Energy Phys. 09 (2020) 176.

[7] L. Donnay, A. Puhm, and A. Strominger, Conformally soft photons and gravitons, J. High Energy Phys. 01 (2019) 184.

[8] S. Banerjee, Symmetries of free massless particles and soft theorems, Gen. Relativ. Gravit. 51, 128 (2019).

[9] S. Stieberger and T. R. Taylor, Symmetries of celestial amplitudes, Phys. Lett. B 793, 141 (2019).

[10] Y. A. Law and M. Zlotnikov, Poincar constraints on celestial amplitudes, J. High Energy Phys. 03 (2020) 085; Erratum, J. High Energy Phys. 04 (2020) 202.

[11] S. Pasterski and S.-H. Shao, Conformal basis for flat space amplitudes, Phys. Rev. D 96, 065022 (2017).

[12] Y. A. Law and M. Zlotnikov, Massive spinning bosons on the celestial sphere, J. High Energy Phys. 06 (2020) 079.

[13] L. Iacobacci and W. Mück, Conformal primary basis for Dirac spinors, Phys. Rev. D 102, 106025 (2020).

[14] S. A. Narayanan, Massive celestial fermions, arXiv:2009 .03883 .

[15] C. Cheung, A. de la Fuente, and R. Sundrum, 4D scattering amplitudes and asymptotic symmetries from 2D CFT, J. High Energy Phys. 01 (2017) 112.

[16] S. Pasterski, S.-H. Shao, and A. Strominger, Gluon amplitudes as 2d conformal correlators, Phys. Rev. D 96, 085006 (2017).

[17] C. Cardona and Y.-t. Huang, S-matrix singularities and CFT correlation functions, J. High Energy Phys. 08 (2017) 133.

[18] A. Schreiber, A. Volovich, and M. Zlotnikov, Tree-level gluon amplitudes on the celestial sphere, Phys. Lett. B 781, 349 (2018).

[19] S. Stieberger and T. R. Taylor, Strings on celestial sphere, Nucl. Phys. B935, 388 (2018).

[20] D. Nandan, A. Schreiber, A. Volovich, and M. Zlotnikov, Celestial amplitudes: Conformal partial waves and soft limits, J. High Energy Phys. 10 (2019) 018.

[21] M. Pate, A.-M. Raclariu, and A. Strominger, Conformally soft theorem in gauge theory, Phys. Rev. D 100, 085017 (2019).

[22] T. Adamo, L. Mason, and A. Sharma, Celestial amplitudes and conformal soft theorems, Classical Quantum Gravity 36, 205018 (2019).

[23] A. Puhm, Conformally soft theorem in gravity, J. High Energy Phys. 09 (2020) 130.

[24] W. Fan, A. Fotopoulos, S. Stieberger, and T. R. Taylor, On Sugawara construction on celestial sphere, J. High Energy Phys. 09 (2020) 139.
[25] S. Banerjee, S. Ghosh, and P. Paul, MHV graviton scattering amplitudes and current algebra on the celestial sphere, arXiv:2008.04330.

[26] W. Fan, A. Fotopoulos, and T. R. Taylor, Soft limits of Yang-Mills amplitudes and conformal correlators, J. High Energy Phys. 05 (2019) 121.

[27] A. Fotopoulos and T. R. Taylor, Primary fields in celestial CFT, J. High Energy Phys. 10 (2019) 167.

[28] A. Fotopoulos, S. Stieberger, T. R. Taylor, and B. Zhu, Extended BMS algebra of celestial CFT, J. High Energy Phys. 03 (2020) 130.

[29] M. Pate, A.-M. Raclariu, A. Strominger, and E. Y. Yuan, Celestial operator products of gluons and gravitons, arXiv: 1910.07424.

[30] S. Banerjee, S. Ghosh, and R. Gonzo, BMS symmetry of celestial OPE, J. High Energy Phys. 04 (2020) 130.

[31] Y. A. Law and M. Zlotnikov, Relativistic partial waves for celestial amplitudes, arXiv:2008.02331.

[32] E. Casali and A. Puhm, A double copy for celestial amplitudes, arXiv:2007.15027.

[33] S. Albayrak, C. Chowdhury, and S. Kharel, On Loop Celestial Amplitudes for Gauge Theory and Gravity, arXiv: 2007.09338 [Phys. Rev. Lett. (to be published)].

[34] N. Banerjee, S. Banerjee, S. Atul Bhatkar, and S. Jain, Conformal structure of massless scalar amplitudes beyond tree level, J. High Energy Phys. 04 (2018) 039.

[35] Z. Bern, A. De Freitas, L. J. Dixon, and H. Wong, Supersymmetric regularization, two loop QCD amplitudes and coupling shifts, Phys. Rev. D 66, 085002 (2002).

[36] A. Strominger, Celestial Holography, Talk delivered at Strings 2020 (Cape Town, South Africa, 2020).

[37] Z. Bern, L. J. Dixon, and V. A. Smirnov, Iteration of planar amplitudes in maximally supersymmetric Yang-Mills theory at three loops and beyond, Phys. Rev. D 72, 085001 (2005).

[38] C. Anastasiou, Z. Bern, L. J. Dixon, and D. Kosower, Planar Amplitudes in Maximally Supersymmetric Yang-Mills Theory, Phys. Rev. Lett. 91, 251602 (2003).

[39] L. F. Alday and J. M. Maldacena, Gluon scattering amplitudes at strong coupling, J. High Energy Phys. 06 (2007) 064.

[40] H. A. González and F. Rojas (unpublished).

[41] S. Badger, Automating QCD amplitudes with on-shell methods, J. Phys. Conf. Ser. 762, 012057 (2016).

[42] Z. Bern and D. A. Kosower, The computation of loop amplitudes in gauge theories, Nucl. Phys. B379, 451 (1992).

[43] Z. Bern, String based perturbative methods for gauge theories, in Theoretical Advanced Study Institute (TASI 92): From Black Holes and Strings to Particles (Boulder, 1992), Vol. 6, pp. 0471-536.

[44] Z. Bern, G. Chalmers, L. J. Dixon, and D. A. Kosower, One Loop N Gluon Amplitudes with Maximal Helicity Violation Via Collinear Limits, Phys. Rev. Lett. 72, 2134 (1994).

[45] G. Mahlon, Multi-gluon helicity amplitudes involving a quark loop, Phys. Rev. D 49, 4438 (1994).

[46] G. Mahlon, One loop multi-photon helicity amplitudes, Phys. Rev. D 49, 2197 (1994).

[47] Z. Bern and A. Morgan, Massive loop amplitudes from unitarity, Nucl. Phys. B467, 479 (1996). 
[48] H. Hannesdottir and M. D. Schwartz, A finite $S$-matrix, arXiv:1906.03271.

[49] H. Hannesdottir and M. D. Schwartz, S-Matrix for massless particles, Phys. Rev. D 101, 105001 (2020).

[50] J. M. Henn and J. C. Plefka, Scattering Amplitudes in Gauge Theories (Springer, Berlin, 2014), Vol. 883.

[51] M. B. Green, J. H. Schwarz, and L. Brink, $N=4$ YangMills and $N=8$ supergravity as limits of string theories, Nucl. Phys. B198, 474 (1982).

[52] C. Schubert, Perturbative quantum field theory in the string inspired formalism, Phys. Rep. 355, 73 (2001).

[53] F. Cachazo, M. Spradlin, and A. Volovich, Hidden beauty in multiloop amplitudes, J. High Energy Phys. 07 (2006) 007.

[54] M. Spradlin, Multiloop gluon amplitudes and AdS/CFT, eConf C0706044, 05 (2007), https://www.slac.stanford.edu/ econf/C0706044/pdf/05.pdf.

[55] V. A. Smirnov, Analytical result for dimensionally regularized massless on shell double box, Phys. Lett. B 460, 397 (1999).
[56] Z. Bern, M. Czakon, L. J. Dixon, D. A. Kosower, and V. A. Smirnov, The four-loop planar amplitude and cusp anomalous dimension in maximally supersymmetric Yang-Mills theory, Phys. Rev. D 75, 085010 (2007).

[57] M. Srednicki, Quantum Field Theory (Cambridge University Press, Cambridge, England, 2007).

[58] A. Fotopoulos, S. Stieberger, T. R. Taylor, and B. Zhu, Extended super BMS algebra of celestial CFT, J. High Energy Phys. 09 (2020) 198.

[59] Z. Bern, D. C. Dunbar, and T. Shimada, String based methods in perturbative gravity, Phys. Lett. B 312, 277 (1993).

[60] D. C. Dunbar, J. H. Ettle, and W. B. Perkins, Augmented recursion for one-loop gravity amplitudes, J. High Energy Phys. 06 (2010) 027.

[61] D. J. Gross and P. F. Mende, The high-energy behavior of string scattering amplitudes, Phys. Lett. B 197, 129 (1987).

[62] D. J. Gross and P. F. Mende, String theory beyond the Planck scale, Nucl. Phys. B303, 407 (1988).

[63] D. J. Gross and J. Manes, The high-energy behavior of open string scattering, Nucl. Phys. B326, 73 (1989). 